\title{
Sinop Arkeoloji Müzesi Yeni Yazıtlar I
}

\author{
New Inscriptions from Sinop Archaelogical Museum I \\ Hüseyin Sami ÖZTÜRK - Fuat DERELI
}

open access journals

PHASELIS: Disiplinlerarası Akdeniz Araştırmaları Dergisi'nde bulunan içeriklerin tümü kullanıcılara açık, serbestçe/ücretsiz "açık erişimli" bir dergidir. Kullanıcılar, yayıncıdan ve yazar(lar)dan izin almaksızın, dergideki makaleleri tam metin olarak okuyabilir, indirebilir, dağıtabilir, makalelerin çıktısını alabilir ve kaynak göstererek makalelere bağlantı verebilir.

PHASELIS: Disiplinlerarası Akdeniz Araştırmaları Dergisi uluslararası hakemli elektronik (online) bir dergi olup değerlendirme süreci biten makaleler derginin web sitesinde (journal.phaselis.org) yıl boyunca ilgili sayının içinde (Volume I: Ocak-Aralık 2015) yayımlanır. Aralık ayı sonunda ilgili yıla ait sayı tamamlanır.

Dergide yayımlanan eserlerin sorumluluğu yazarlarına aittir. 


\title{
Sinop Arkeoloji Müzesi Yeni Yazıtlar I \\ New Inscriptions from Sinop Archaeological Museum I
}

\author{
Hüseyin Sami ÖZTÜRK* Fuat DERELi**
}

Öz: Sinop Arkeoloji Müzesi'nden alınan bir izinle müzedeki yazıtlar kayıt altına alınmış ve üzerinde çalışımıştır. Bütün yazıtlar Sinop'tan bulunmuş olup, antik Sinope kentine aittir. Bu makalede müzede gerçekleştirilen epigrafik çalışmanın ilk sonucu olarak yedi yeni yazıt tanıtılmaktadır.

Anahtar sözcükler: Sinope · Pontos/Pontus · Hadrianus · Yazıt · Klasik Dönem · Geç Antikçağ · Diakon

Abstract: The inscriptions in the Sinop Archaeological Museum were copied and studied with the permission of the directorship of the Sinop Archaeological Museum. These inscriptions were all found within the vicinity of Sinop and belong to the ancient city of Sinope. In this article providing the first results of epigraphical work in the museum, seven previously unpublished Greek inscriptions are presented.

Keywords: Sinope $\cdot$ Pontos/Pontus · Hadrianus · Inscription · Classical Period · Late Antiquity · Diacon

2009 yılından itibaren, Sinop Arkeoloji Müzesi Müdürlüğü'nden alınan bir izinle, müzede yayımlanmış ve yayımlanmamış tüm yazıtların yeni bir corpus altında toplanması için bir proje başlatılmıştır. Bu kapsamda, Sinop başta olmak üzere civardan müzeye getirilen tüm yazıtlar tarafımızdan yeniden kayıt altına alınmıştır. Yapılan bu çalışmanın ardından yayımlanmamış yeni birçok yazıt tespit edilmiştir. Şimdiye kadar Sinop Arkeoloji Müzesi ve Sinop'tan yazıtların bir araya getirildiği en kapsamlı eser D. French tarafından 2001 yılında yayımlanan I.Sinope adlı çalışmadır. Bu çalışma dışında 2005 yılında L. Ruscu, Sinop'tan bir yazıt yayımlamıştır. Müze'deki çalışmada birçok farklı türden ve dönemden yazıt kayıt altına alınmıştır. Bu makalede, aşağıda görüleceği üzere bu yazıtlardan bir kısmı bilim dünyasına tanıtılmaktadır. Yazıtların büyük çoğunluğu Roma İmparatorluk Dönemi'ne ait olup, mezar yazıtları ağılıktadır. Makalemizde, biri Klasik Dönem mezar yazıtı olmak üzere, toplam 6 mezar yazıtı ile İmparator Hadrianus için yapılan bir onur kaidesinin sadece üst kısmına ait yazıt yer almaktadır.

\section{Hekataios'un Mezarı}

Kireçtaşından, minyatür, Dor stili sütun biçiminde bir mezar steli. Sinop Arkeoloji Müzesi bahçesinde sergilenmektedir (Müze Env. No: 12.01.003).

Buluntu Yeri: Sinop ili Bostancılı Köyü. Satın alma yoluyla Müze'ye kazandırılmıs.

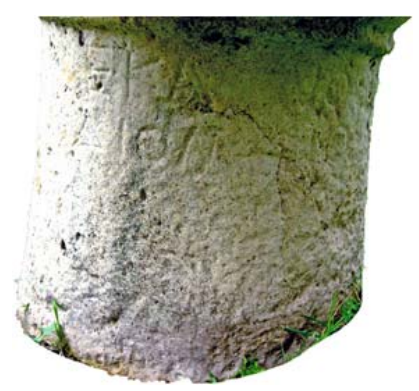

* Yrd. Doç. Dr., Marmara Üniversitesi, Fen-Edebiyat Fakültesi, Tarih Bölümü, Eskiçağ Tarihi Anabilim Dalı, İstanbul.hsoztrk@yahoo.com.

** Arkeolog, Sinop Arkeoloji Müzesi Müze Müdür Vekili, Sinop. 
Ölçüler: Y: 51 cm.; HY: ca. 4,6 cm.

Kayıt Bilgileri: Env. No: 13; Kayıt: H. S. Öztürk; Foto: H. S. Öztürk; Kalıp: -

Tarih: M.Ö. V-IV. yüzyıI

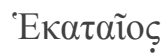 \\ $\Delta$ tovvó́ov. \\ Dionysios oğlu Hekataios (burada yatıyor).
}

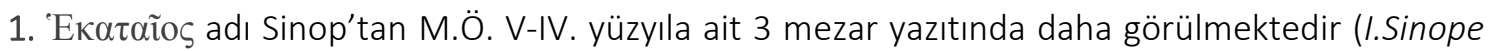
19; 20 (iki); 64). Bu ismin Pontos Bölgesi ile Küçük Asya'da görüldüğü yerler için ayrıca bk. LGPN

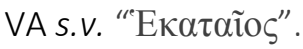

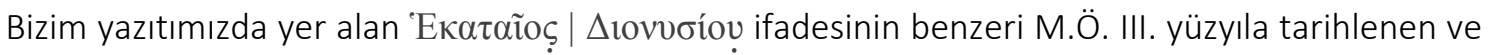

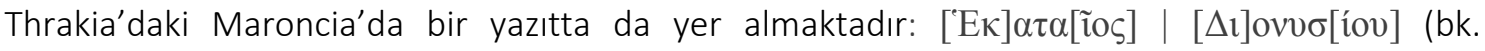
I.AegThrace 232).

\title{
2. İmparator Hadrianus'un Onurlandırılması
}

Mermerden sol üst tarafı kısmen; altı ise tamamen kırık silindirden bir onur kaidesi. Silindirin üstünde iki adet zıvana yuvası bulunmaktadır. Şimdi Sinop Arkeoloji Müzesi bahçesinde sergilenmektedir (Müze Env. No: 17.1.07).

Buluntu Yeri: Sinop ili.

Ölçüler: Y: 70 cm.; Ç: 69 cm. (üst), 60 cm. (alt); HY: 5,4-8,6 cm. Kayıt Bilgileri: Env. No: 3; Kayıt: H. S. Öztürk; Foto: H. S. Öztürk; Kalıp: -

Tarih: M.S. 98-117.

$$
\text { Imp(eratori) Caesari }
$$

2 divi Traiani

Parthici fil(io) divi Nervae

4 [nepoti Traiano Hadriano Augusto pontifici maximo, tribunicia potestate

6

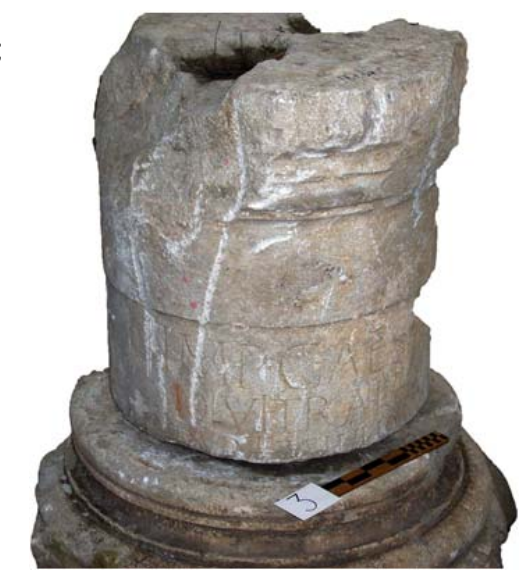

(Bu heykeli) Tanrısal Nerva'nın torunu, Tanrısal Particus Traianus'un oğlu, pontifex maximus, tribunicia potestate'sinin (falanca yılında) bulunan imparator Caesar Traianus Hadrianus Augustus için (dikti/ler.)

Yazıt Hadrianus'un Sinop antik kentinde onurlandırılmasına ilişkin ilk örnektir. Yazıtın geri kalan kısmı kırık olduğu için, Hadrianus'un kim/kimler veya hangi kurum tarafından onurlandırıldığını bilemiyoruz.

\section{Iulia Eunome'nin Mezarı}

Mermerden, dikdörtgen formda bir mezar steli. Üst kısmı kırık. Şimdi Sinop Arkeoloji Müzesi Bahçesi'nde sergilenmektedir. (Müze Env. No: 8. 2001).

Buluntu Yeri: Sinop ili.

Ölçüler: Y: 1,15 m.; G: $57 \mathrm{~cm}$.; D: $32 \mathrm{~cm} . ; H Y: 6-7 \mathrm{~cm}$.

Kayıt Bilgileri: Env. No: 14; Kayıt: H. S. Öztürk; Foto: H. S. Öztürk; Kalıp: - 
Tarih: M.S. I-II. yüzyII.

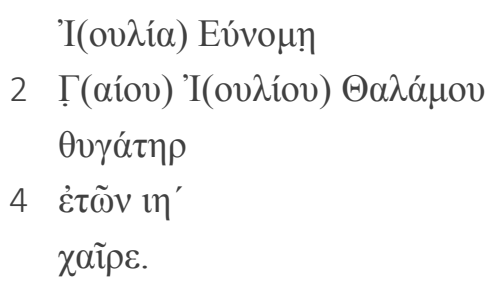

\section{3 ӨYГАТНP lapis | 5 XAIPE lapis.}

18 yıl yaşamış olan, Gaius Iulius Thalamos kızı Iulia Eunome (burada yatıyor). Hoş̧̧akalın!

1. Ev́voun = Eủvón. Eủvón formu için bk. Syrakusai/Sicilia (SEG 38, 969).

2. $\Theta \alpha ́ \lambda \alpha \mu o \varsigma$ adının örnekleri için bk. Uşak/Lydia (Varinlioğlu 1983, 78-80 no: 2 = SEG 33, 1119), Uşak/Lydia (Varinlioğlu

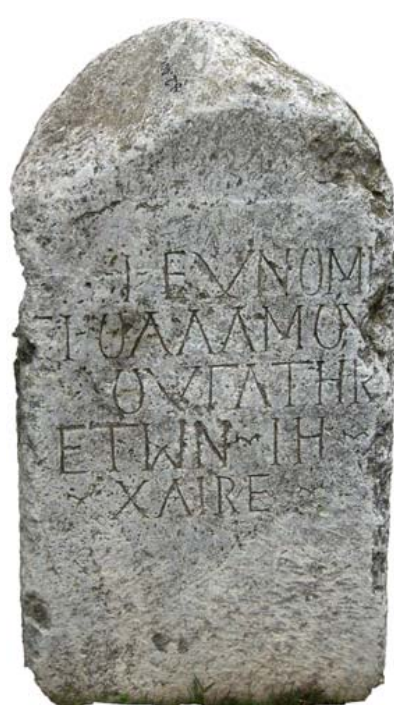
1989, 30 no. 16 = SEG 39, 1309), Silandos/Lydia (Hermann - Malay 2007, 101 vd. no. $73=$ SEG 57, 1225), Neapolis/Pisidia (Labarre et al. 2007, 142 no. 2 = SEG 57, 1404).

\section{Gaius Longidius Niger'in Mezarı}

Dikdörtgen prizmal gövdeli, üçgen alınlıklı, tepe ve yan akroterli, kaide kademeli mermerden stel. Gövde üst kısma yakın bir noktada kırılmış olup stelin ön yüzünde kilit, sadak, rulo, rozet gibi kabartmalar mevcut. Stel, hibe yoluyla Sinop Müzesi'ne kazandırılmış olup, müze bahçesinde sergilenmektedir (Müze Env. No: 7.1.007).

Buluntu Yeri: Sinop II Merkezi, Gelincik Mahallesi.

Ölçüler: Y: 1,51 m.; G: 59 cm.; D: 30 cm.; HY: 3,4-4,3 cm.

Kayıt Bilgileri: Env. No: 2; Kayıt: H. S. Öztürk; Foto: H. S. Öztürk; Kalıp: -

Tarih: M.S. I-II. yüzyıl.

C(aius) Longidius C(aii) f(ilius)

Niger ann(O) XXIII

Gaius oğlu, 23 yaşında ölen Gaius Longidius Niger

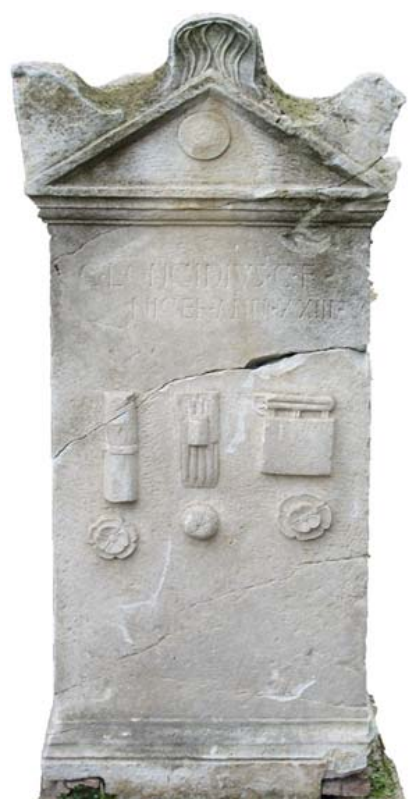
(burada yatıyor).

1 Longidius adı Bithynia'dan Klaudiupolis antik kentinde veteranus L. Valerius Longidius'a ait bir mezar yazıtından bilinmektedir (I.Klaudiupolis 162).

\section{Lucia Petronia Tertulla'nın Mezarı}

Kireçtaşından bir lahit teknesinin ön yüzüne ait bir fragman. Sağ alt kenar ile sol kenar tamamen kırıktır. 2009 yılında Sinop Arkeoloji Müzesi'ne getirilmiş olup; müze bahçesinde sergilenmektedir.

Buluntu Yeri: Sinop antik kentinin denize bakan sur duvarındaki dolgunun içinde bulunmuş.

Ölçüler: Y: 53 cm.; G: 90 cm.; D: 12 cm.; HY: 3-5 cm. 
Kayıt Bilgileri: Env. No: 5; Kayıt: H. S. Öztürk; Foto: H. S. Öztürk; Kalıp: -

Tarih: M.S. I-II. yüzyıl.

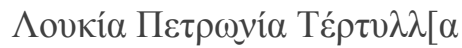

Filanca kızı(?) Lucia Petronia Tertulla

(burada yatıyor).

Mezar sahibinin taşıdığı isminden dolayı bir Roma

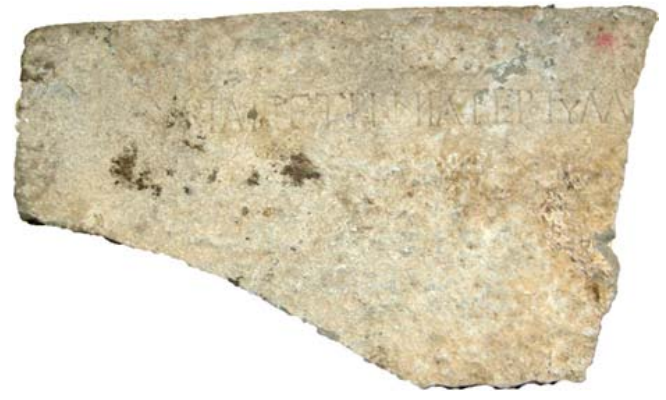
vatandaşı olduğu anlaşılmaktadır.

\section{Helios ile Theopropos'un Mezarı}

Kireçtaşından, sol alt kenarı kısmen kırılmış ve ortası çukurlaştırılmış bir stel. Yan ve arka yüzler kaba bırakılmıştır. Müze bahçesinde sergilenmektedir (Müze Env. No. 7.2.001).

Buluntu Yeri: Sinop il Merkezi.

Ölçüler: Y: 77 cm.; G: $41 \mathrm{~cm}$.; D: $22 \mathrm{~cm}$.; HY: 3,5-7 cm.

Kayıt Bilgileri: Env. No: 8; Kayıt: H. S. Öztürk; Foto: H. S. Öztürk; Kalıp: -

Tarih: M.S. I-II. yüzyıl.

$$
\begin{aligned}
& \text { "H } \lambda \text { ios } \\
& 2 \text { Z } \omega \tilde{i} \lambda- \\
& \text { ov } \chi- \\
& 4\{\chi\} \alpha \text { ĩ } \varepsilon .
\end{aligned}
$$

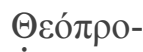

$$
\begin{aligned}
& 6 \pi \text { os } \mathrm{H} \lambda \mathrm{i}^{-} \\
& \text {ov }
\end{aligned}
$$

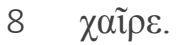

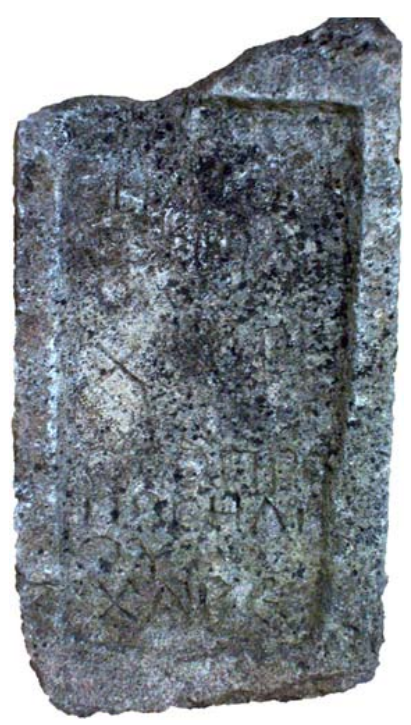

3 v.dd. XXAIPE lapis.

Zoilos oğlu Helios (burada yatıyor). Hoşçakalın!

Helios oğlu Theopropos (da burada yatıyor).

Hoş̧̧akalın!

5 v.d. $\Theta \varepsilon o ́ \pi \rho o \pi \circ \varsigma$ adı Sinop’tan bilinmektedir. Bu adın Sinop ve Küçük Asya'daki örnekleri için bk. LGPN VA; VB s.V. “ $\Theta \varepsilon \delta ́ \pi \rho o \pi \circ \varsigma^{\prime}$.

Yazıttan, mezarın baba ile oğluna ait olduğu anlaşılmaktadır. Yazıtın ilk satırında adı geçen kişi mezarın asıl sahibi olmalıdır ki, bu kişi Helios'tur. Daha sonra, Helios bu mezarı oğlu Theopropos için de kullanıma açmış görünmektedir.

\section{Diakon Thomas'ın Mezarı}

Kireçtaşından ya da bölgeye özgü kumtaşı adı verilen malzemeden yapılmış, bir mezara ait parça. Günümüze sağlam olarak ulaşmıştır. Sinop Arkeoloji Müzesi Bahçesi'nde sergilenmektedir.

Buluntu Yeri: Sinop Merkez İlçe, Erfelek/Hasandere Köyü. 
Ölçüler: Y: 1,11 m.; G: 1,23 m.; D: 80 cm.; HY: 2-7 cm.

Kayıt Bilgileri: Env. No: 7; Kayıt: H. S. Öztürk; Foto: H. S. Öztürk; Kalıp: -

Tarih: M.S. IV-V. yüzyıl.

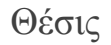

vacat

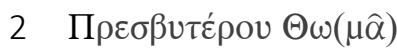

$\delta 1 \alpha \kappa \omega v$

vacat

vacat

4 हỉ $\pi 1 \varsigma \theta \varepsilon \lambda \eta ́ \sigma 1 \pi \alpha \rho \alpha-$

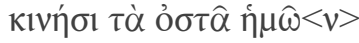

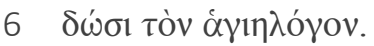

$5 \mathrm{HM} \Omega$ lapis.

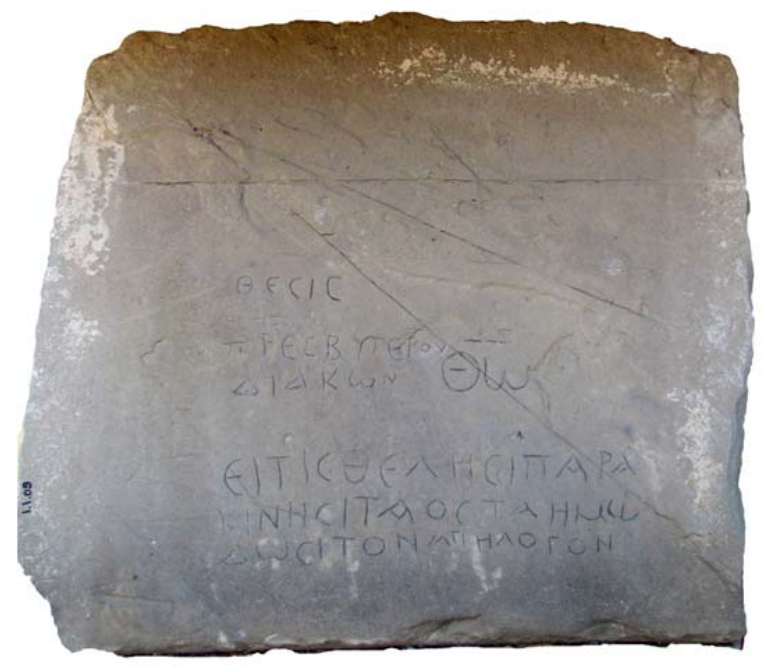

Presbyteros (ve) Diakon Thomas'ın istirahatgâhı.

Eğer birisi kemiklerimizi kaldırmak isterse, hesabını verecektir.

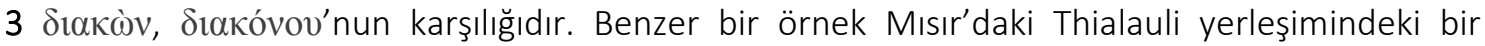

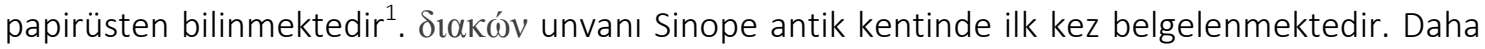

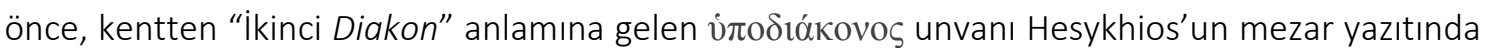
bilinmekteydi (I.Sinope 190).

Yazının harf karakterleri oldukça tahrip olmuş bir durumda olup, satıhlar zayıftır. Söz konusu yazıta göre, mezar diakon Thomas'a aittir. Ancak yazıtta, "birisi kemiklerimizi kaldırmak isterse" ifadesinden mezarda, sadece diakon Thomas değil; olasılıkla O'nun izin verdiği kişilerin de yattığı anlaşılabilir.

Kaynak:http://www.trismegistos.org/geo/georef.php?tm=2395\&token=7\&total=12\&order=date_y1,date_y2 


\section{BIBBLIYOGRAFYA}

Herrmann - Malay 2007

I.AegThrace

I. Klaudiupolis

I.Sinope

Labarre et al. 2007

LGPN VA

LGPN VB

Ruscu 2005

SEG

Varinlioğlu 1983

Varinlioğlu 1989
P. Hermann - H. Malay, New Documents from Lydia. Wien 2007.

Epigraphes tes Thrakes tou Aigaiou: Metaxy ton Potamon Nestou kai Hevrou (Nomoi Xanthes, Rhodopes kai Hevrou). Eds. L. D. Loukopoulou, M. G. Parissaki, S. Psoma, A. Zournatzi - D. Triantaphyllo. Athens 2005.

Die Inschriften von Klaudiu Polis, mit einem Anhang von Wolfram Weiser: "Zur Münzprägung von Bithynion". Ed. F. Becker-Bertau. Bonn 1986.

The Inscriptions of Sinope. Ed. D. H. French. Bonn 2004.

G. Labarre, M. Özsait - N. Özsait, "Sites et Inscriptions de la Plaine Cillanienne". Anatolia Antiqua 15 (2007) 113-146.

"A Lexicon of Greek Personal Names". Volume VA: Coastal Asia Minor: Pontos to Ionia. Ed. T. Corsten. Oxford 2010.

"A Lexicon of Greek Personal Names". Volume VB: Coastal Asia Minor: Caria to Cilicia. Eds. J.-S. Balzat, R. W. V. Catling, É. Chiricat, F. Marchand T. Corsten. Oxford 2013.

L. Ruscu, "Two Pontic Notes". EA 38 (2005) 125-130.

Supplementum Epigraphicum Graecum.

E. Varinlioğlu, "Zeus Orkamaneites and the Expiatory Inscriptions". EA 1 (1983) 75-87.

E. Varinlioğlu, "Die Inschriften aus dem Museum von Uşak". EA 13 (1989) 17-36. 